Lepr Rev (1987) 58, 173-177

SPECIAL ARTICLE

\title{
Field detection of early neuritis in leprosy
}

\author{
E P FRITSCHI \\ Schieffelin Leprosy Research and Training Centre, Karigiri, PIN: \\ 632106 NA District, Tamil Nadu, S. India
}

\section{Accepted for publication 26 January 1986}

Summary Five field tests for detecting early damage to peripheral mixed nerve trunks in leprosy, are described. The regular monthly use of these tests will assist in reducing deformity. The whole set can be administered in less than ten seconds.

Neuritis in leprosy is frequently the precursor of irreversible sensory and motor loss. But, if the condition is detected early enough, deformity can be averted.

Unfortunately the term 'Neuritis' is usually associated with pain or tenderness. Srinivasan ${ }^{3}$ has pointed out that in his study, 47 out of 58 patients $(81 \%)$ gave no significant history of pain or tenderness in the affected nerves. This is also the experience of two other workers. ${ }^{1,2}$

It is therefore not sufficient to be only on the look-out for painful tender nerves. One of the most reliable signs of early neuritis is some degree of loss of function.

It is not necessary to have a detailed knowledge of the anatomy of the extremities, in order to detect early weakness of muscles, and correctly attribute this to the particular nerve involved.

It is not possible to spend a lot of time grading muscles on a quantitative scale in the field. There is not enough time for any of the field clinic staff to do this.

Theref ore a set of easy tests have been devised which aims at revealing early weakness of groups of muscles.

There are only six mixed nerve trunks commonly affected in leprosy. To reveal early weakness in any of these, on either side, using the following tests, will take less than 10 seconds. When weakness is suspected the field worker must refer the patient immediately to the referral centre for detailed motor and sensory evaluation of nerve function, and early treatment with steroids, if the muscle weakness is confirmed.

\section{Test 1 Facial nerves}

The patient is asked to close both eyes (Figure 1). Any delayed or incomplete closure is suggestive of facial nerve involvement. 


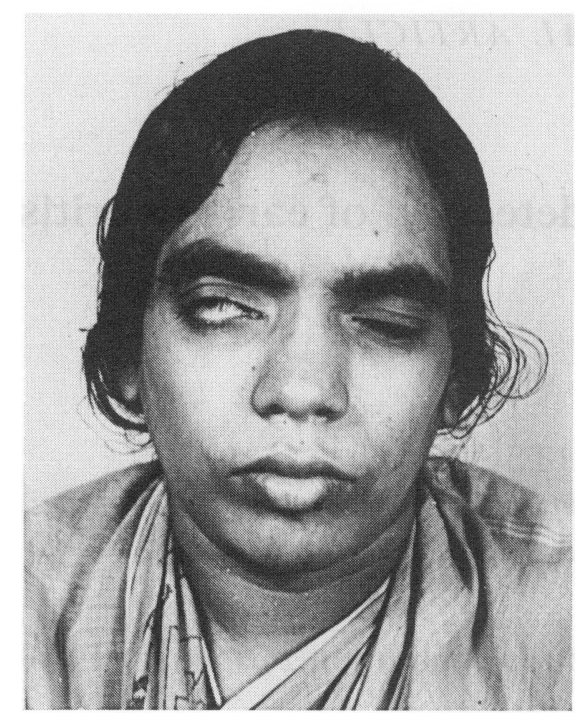

Figure 1. Patient with one eye lagophthalmos attempting to close both eyes. Note right eye paresis.
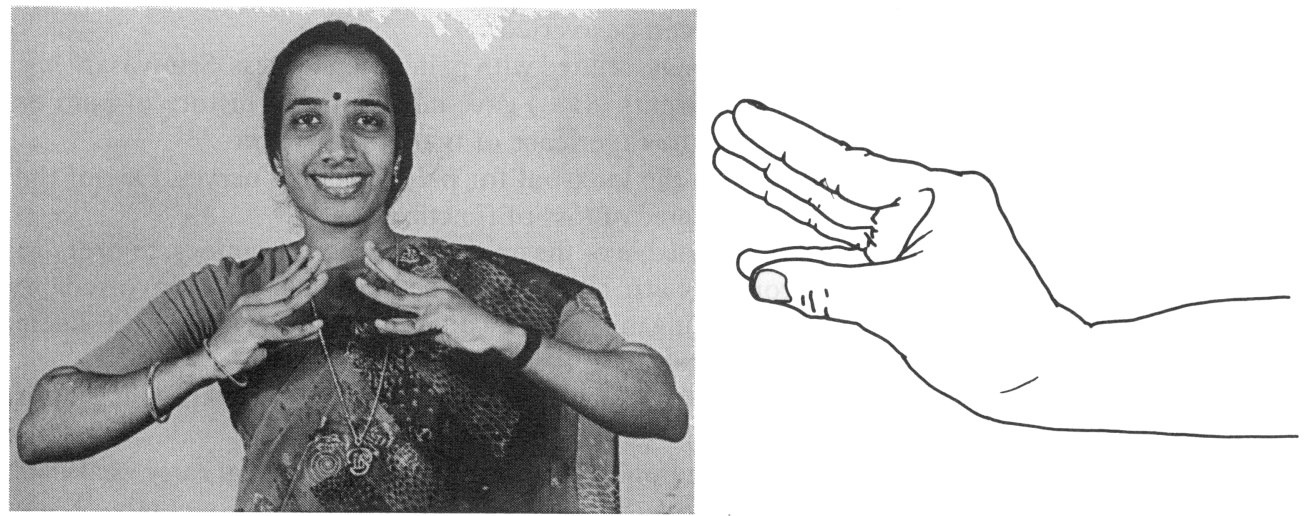

Figure 2. The Indian Classical dance pose which tests all three nerves of the forearm and hand. Dorsiflexion of wrist, radial nerve; extension of fingers, ulnar nerve; and abduction of thumb, median nerve.

Figure 3. Diagrammatic representation of the ulna-median radial gesture viewed from the radial side. 

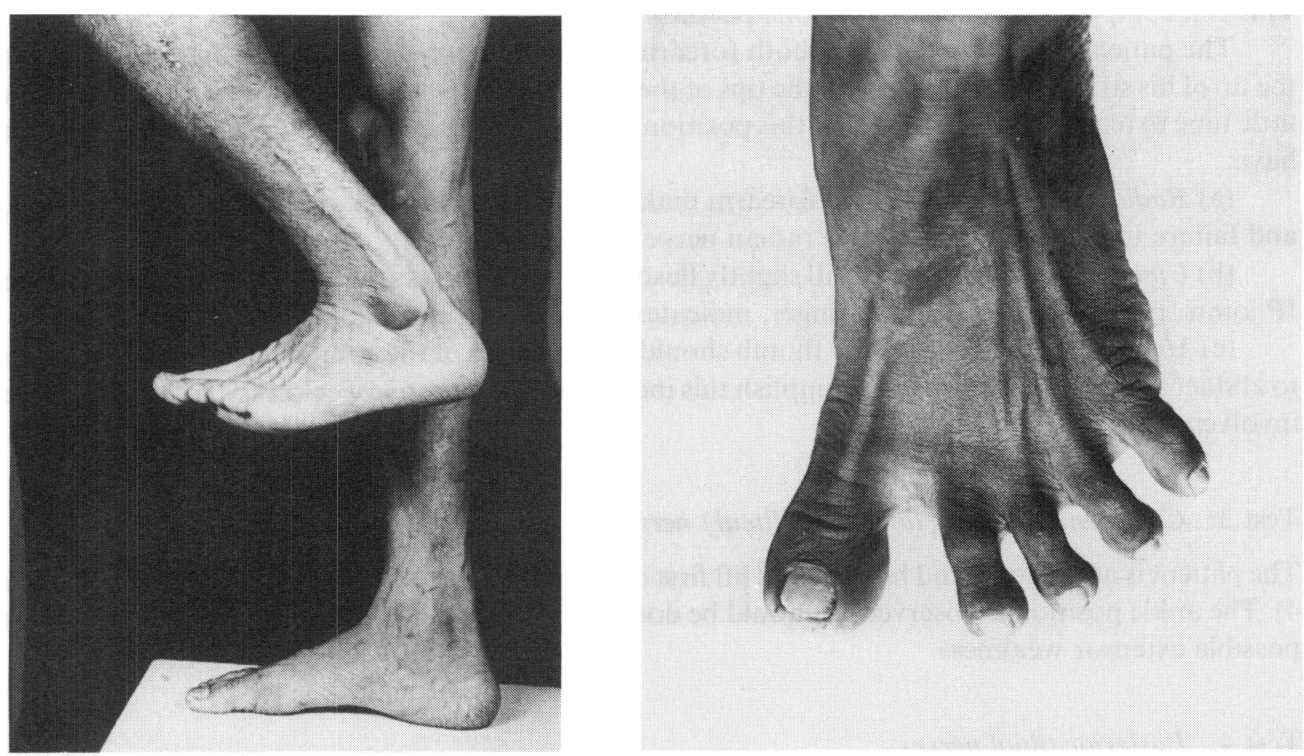

Figure 4. Test for lateral popliteal nerve paralysis. Note ankle and toe dorsiflexion.

Figure 5. Spreading of toes, test for intrinsic muscles of the foot-posterial tibial nerve.

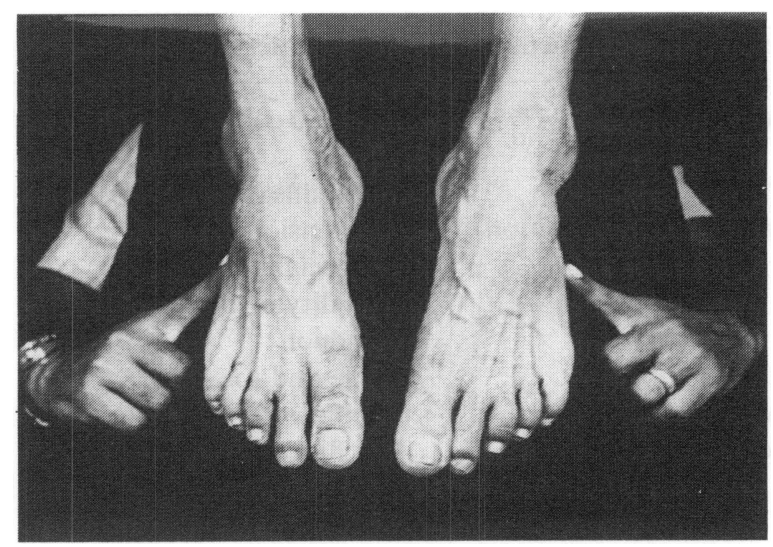

Figure 6. The sensory test for posterial tibial nerve paralysis. 


\section{Test 2 Ulna median and radial nerves}

This is a composite test achieved in one position (Figure 2).

The patient is asked to pronate both forearms, raise both wrists, extend all fingers and touch the tip of his straight little finger with the tips of the thumbs of the same hand (Figure 3). It takes very little time to teach an illiterate patient this position. Analysing this in terms of the nerves involved we have:

(a) Radial nerve. The pronated forearm makes the wrist extension an antigravity movement, and failure to achieve this indicates radian nerve involvement.

(b) Ulna nerve. The fingers are all slightly flexed at the MP joints and failure to straighten all the IP joints, particularly in the little finger, indicates weakness of ulna supplied muscles.

(c) Median nerve. The tip of the thumbshould touch the tip of the straight little finger. Inability to abduct and flex the thumb to accomplish this indicates thenar muscle weakness and median nerve involvement.

\section{Test 3 Common peroneal (lateral popliteal) nerves}

The patient is asked to extend his toes and lift first one and then the other foot off the ground (Figure 4). The ankle position is observed. It should be dorsiflexed. Inability to dorsiflex the ankle indicates possible extensor weakness.

\section{Test 4 Posterior tibial nerves}

This is the only one which may present some difficulty. The patient is asked to remove his slippers and spread his toes (Figure 5). If he cannot do that (and some normal persons especially in a shoewearing population have difficulty), then the observer may gently stroke the lateral border of both feet together (Figure 6) and ask 'Do you feel this normally and the same on each side?'. Weakness of

Table 1. Below is a summary of Tests $1-4$

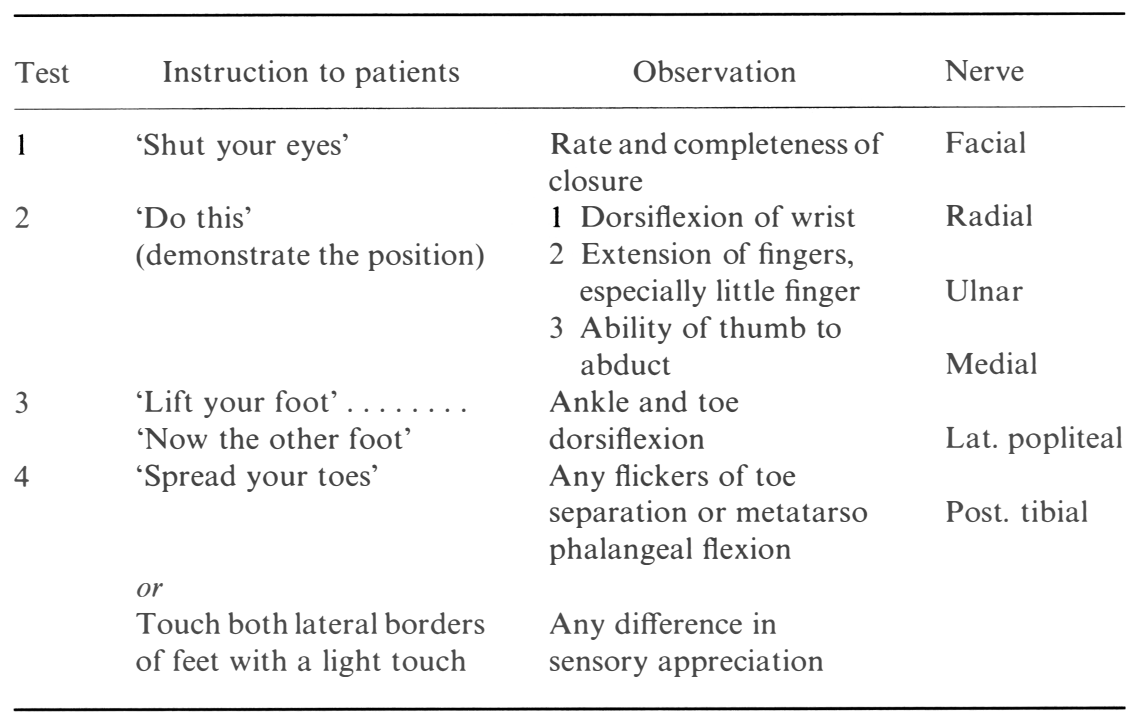


the intrinsic muscles of the foot predisposes to forefoot ulceration due to undue prominence of the metatarsal heads on the plantar surface.

Even a busy field worker can afford the 10 seconds it takes to carry out these tests.

It is important that he should apply these tests to patients who have had no obvious nerve damage, especially to multiple lesion cases of borderline leprosy.

A word to the doctor in the referral centre. Never discourage or ridicule your field worker if he refers a case who, on detailed examination, turns out not to have any nerve damage. The early detection of nerve damage is essential if we are to prevent deformity. Early detection depends on early suspicion by the person in the team who sees the patient first and most regularly. Encourage him!

\section{Acknowledgments}

The author acknowledges with thanks the assistance of several of his patients and staff in modelling the illustrations, to Mr Sanjay Agrawal and Mr Ramadoss for the photographs and Miss Shanthi Sivaprakasam for typing the manuscript.

\section{References}

${ }^{1}$ Fritschi EP. Reconstructive Surgery in Leprosy. John Wright and Sons, p. 11, 1971.

2 Ross FW. Neuritis. In A Manual of Leprosy, 3rd edn, Thangaraj RH, p. 170, The Leprosy Mission, New Delhi, 1983.

3 Srinivasan H, Rao KS, Shanmugam N. Steroid Therapy in Recent "Quiet Nerve Paralysis". Lepr India, 1982; 54: 412-9. 